Maddalena Paganin, Giulia Fabbri, Katia Polato, and Giuseppe Basso, Università di Padova, Padova; Valentino Conter, Ospedale San Gerardo; Maria Grazia Valsecchi, Università di Milano Bicocca, Monza; Elena Barisone and Franca Fagioli, Ospedale Infantile Regina Margherita, Torino; Giovanni Cazzaniga, Università di Milano Bicocca, Milan: Eugenia Giraldi and Maurizio Aricò, Ospedale Papa Giovanni XXIII di Bergamo, Italy

Published online ahead of print at www.jco.org on October 6, 2014.

M.P. and G.F. contributed equally to this work.

Authors' disclosures of potential conflicts of interest and author contributions are found at the end of this article.

Corresponding author: Maurizio Aricò, MD, Direttore Generale, Azienda Sanitaria Provinciale Ragusa, Italy; e-mail: maurizio.arico@ittumori.it.

(C) 2014 by American Society of Clinical Oncology

0732-183X/14/3231w-3553w/\$20.00

DOI: 10.1200/JCO.2014.56.0698

\title{
Postinduction Minimal Residual Disease Monitoring by Polymerase Chain Reaction in Children With Acute Lymphoblastic Leukemia
}

Maddalena Paganin, Giulia Fabbri, Valentino Conter, Elena Barisone, Katia Polato, Giovanni Cazzaniga, Eugenia Giraldi, Franca Fagioli, Maurizio Aricò, Maria Grazia Valsecchi, and Giuseppe Basso

Listen to the podcast by Dr Bhojwani at www.jco.org/podcasts

$$
\text { A } \begin{array}{llllllll}
\text { B } & \text { S } & \text { T } & \text { R } & \text { A } & \text { C } & \text { T }
\end{array}
$$

\section{Purpose}

Acute lymphoblastic leukemia ( $A L L)$ is the most common pediatric cancer. Monitoring minimal residual disease (MRD) by using real-time quantitative polymerase chain reaction (RQ-PCR) provides information for patient stratification and individual risk-directed treatment. Cooperative studies have documented that measurement of blast clearance from the bone marrow during and after induction therapy identifies patient populations with different risk of relapse. We explored the possible contribution of measurements of MRD during the course of treatment.

\section{Patients and Methods}

We used RQ-PCR to detect MRD in 110 unselected patients treated in Italy in the International Collaborative Treatment Protocol for Children and Adolescents With Acute Lymphoblastic Leukemia (AIEOP-BFM ALL 2000). The trial took place in AIEOP centers during postinduction chemotherapy. Results were categorized as negative, low positive (below the quantitative range $\left.\left[<5 \times 10^{-4}\right]\right)$, or high positive $\left(\geq 5 \times 10^{-4}\right)$. Patients with at least one low-positive or high-positive result were assigned to the corresponding subgroup.

\section{Results}

Patients who tested high positive, low positive, or negative had significantly different cumulative incidences of leukemia relapse: $83.3 \%, 34.8 \%$, and $8.6 \%$, respectively $(P<.001)$. Two thirds of positive cases were identified within 4 months after induction-consolidation therapy, suggesting that this time frame may be most suitable for cost-effective MRD monitoring, particularly in patients who did not clear their disease at the end of consolidation.

\section{Conclusion}

These findings provide further insights into the dynamic of MRD and the ongoing effort to define molecular relapse in childhood ALL.

\section{J Clin Oncol 32:3553-3558. (c) 2014 by American Society of Clinical Oncology}

\section{INTRODUCTION}

Acute lymphoblastic leukemia (ALL) is the most common type of cancer in children and adolescents. With current risk-directed treatment approaches, cure rates exceed $80 \% .^{1}$ Leukemia relapse remains the most common cause of treatment failure. Although a proportion of patients may be rescued after relapse, second-line therapy may be ineffective in most of the cases, despite intensive approaches, including allogeneic hematopoietic stem-cell transplantation (HSCT). ${ }^{2,3}$ Minimal residual disease (MRD) monitoring is currently considered the most reliable strategy to evaluate early treatment response and to refine stratification accordingly. ${ }^{4-9}$ Both highly sensitive molecular polymerase chain reaction (PCR) and multiparameter flow cytometry approaches may be used to this purpose in the context of first-line, relapse, or allogeneic HSCT trials. ${ }^{10-17}$ The use of immune gene rearrangements by real-time quantitative PCR (RQ-PCR) allows stratification of approximately $90 \%$ of the patients with a single sensitive marker. ${ }^{17}$

These techniques allow identification of insufficient blast cell clearance during initial treatment with disease persistence and also allow identification of the re-emergence of leukemic cells, thus challenging the criteria for the definition of remission ${ }^{18,19}$ or medullary relapse, which, at this point, are based on standard morphologic evidence of $25 \%$ leukemic blasts in the bone marrow 
(BM) in a patient who previously achieved morphologic complete remission (CR).

Despite adequate technologies that are now widely available, only a few studies have addressed the issue of predicting ALL relapse by prospective, postinduction MRD monitoring. Conversion to MRD positivity during early postconsolidation treatment in adult patients with standard-risk ALL (SR-ALL) was strongly predictive of subsequent morphologic relapse. ${ }^{20}$ Although no interventional strategies have been applied so far in ALL, the experience of the Gruppo Italiano Malattie Ematologiche Maligne dell'Adulto in patients with acute promyelocytic leukemia for which the administration of salvage therapy at the time of molecular relapse improved the chance of rescuing patients ${ }^{21}$ might suggest a potential benefit.

In this study, we addressed this issue by monitoring MRD throughout the treatment program in children with ALL treated with a Berlin-Frankfurt-Münster (BFM) -type intensive chemotherapy.

\section{PATIENTS AND METHODS}

Patients in this study were newly diagnosed with childhood ALL between September 2000 and July 2006 and were enrolled onto the International Collaborative Treatment Protocol for Children and Adolescents With Acute Lymphoblastic Leukemia (AIEOP-BFM ALL 2000). ${ }^{6,10}$ AIEOP-BFM ALL 2000 trial patients from two AIEOP centers were eligible for our study.

\section{Patient Stratification}

The definitions for MRD risk are as follows: SR-MRD: MRD was negative at day 33 (time point 1 [TP1]) and day 78 (TP2) by using at least two molecular targets with a sensitivity $\geq 1 \times 10^{-4}$; intermediate-risk MRD (IR-MRD): MRD was positive at less than $5 \times 10^{-4}$ at TP2; high-risk MRD (HR-MRD): $M R D$ was $\geq 5 \times 10^{-4}$ at TP2. Patients with either prednisonepoor response ( $\geq 1,000$ circulating blasts per microliter on day 8 ) or patients who did not achieve CR after induction phase IA or who had translocation $t(4 ; 11)$ were all allocated to the HR group, independently of MRD results.

\section{Treatment Protocol}

Treatment was administered as reported elsewhere. ${ }^{6,10}$ Briefly, all patients were given a 7-day steroid prephase, induction protocols IA and IB, followed by consolidation with high-dose methotrexate for non-high-risk patients or block therapy for high-risk patients, then by reinduction and by maintenance until 24 months from diagnosis. Treatment details, including randomizations, are provided in the Appendix (online only).

\section{BM Sampling}

All patients underwent surveillance BM aspirate at the beginning of protocol IB, protocol M or first block, protocol II or III, and continuation therapy.

Patients in our study underwent additional surveillance aspirates approximately every 2 months during continuation therapy until month +24 from diagnosis and 2 months after treatment discontinuation. Material from each TP was used for MRD determination. Collected samples were batched and analyzed later on; treating physicians were blinded to the results.

Institutional review board approval for this trial was obtained locally by each participating institution. Informed consent for MRD evaluation on any $\mathrm{BM}$ aspirate was obtained from the parents or legal guardians.

\section{Study Population}

In all, 276 Philadelphia chromosome-negative patients were enrolled onto the AIEOP-BFM ALL 2000 trial in two centers that adopted the additional MRD surveillance. Fifty-five were not eligible for this study because of induction failure $(\mathrm{n}=6)$ or lack of a marker by RQ-PCR with a sensitivity $\geq 10^{-4}(\mathrm{n}=49)$. This proportion of ineligible patients is comparable to that in the overall study. ${ }^{6,10}$ Of 221 eligible patients, 110 accepted surveillance BM sampling.

\section{Statistical Analysis}

$\chi^{2}$ or Fisher's exact tests were used to evaluate the association between the frequency of main characteristics and that of MRD positivity during monitoring. Disease-free survival (DFS) and cumulative incidence of relapse (CIR) were the main end points for outcome analysis. DFS was defined as the time from diagnosis to first treatment failure, which was defined as relapse, death in remission, or development of second malignant neoplasm. Observation of patients was censored at the time of last contact, when no events were observed. The Kaplan-Meier method was used to estimate probabilities of DFS with SEs calculated according to Greenwood's formula and CIR (because no competing events were observed). The Cox regression analysis was performed on the cause-specific hazard of relapse to evaluate the MRD profile after adjusting for risk group. Analyses were carried out by using SAS 9.2 (SAS Institute, Cary, NC).

\section{DNA Isolation}

Mononuclear cells were isolated by Ficoll-Paque gradient centrifugation, and DNA was extracted and purified by using the GentraPuregene DNA Purification Kit (Gentra Systems, Monza, Italy) according to the manufacturer's instructions.

\section{Identification of PCR Targets and Design of Allele-Specific Oligonucleotides}

Genomic DNA samples obtained at diagnosis and at relapse were screened for clonal immunoglobulin $\mathrm{H} / \mathrm{K}$ chain, T-cell receptor gene rearrangements, and SIL-TAL by using published primer sets. ${ }^{17,22,23}$ Clonal immune gene rearrangements were identified by heteroduplex analysis and sequenced by using the BigDye Terminator Cycle Sequencing Kit with the ABIPrism 310 Genetic Analyzer (Applied Biosystems, Foster City, CA). After sequencing, allele-specific oligonucleotides were designed for each PCR target based on the sequence data of the junctional region by using Primer Express software v3.0 (Applied Biosystems).

\section{Evaluation and Interpretation of MRD RQ-PCR Results}

The designed allele-specific oligonucleotides were then tested in combination with germ-line primers and TaqMan probes by RQ-PCR using the 7900HT Sequence Detection System (Applied Biosystems). PCR analysis was then performed and results were interpreted according to the guidelines developed by the European Study Group on MRD Detection in ALL (Euro$\mathrm{MRD}$ ) to reduce the risk of false-negative and false-positive results. ${ }^{24}$ Briefly, three replicates were performed for MRD analysis for each TP; $500 \mathrm{ng}$ of DNA per $25 \mu \mathrm{L}$ was used for each reaction; six replicates of the polyclonal control were used to define the background amplification. Although two informative markers were mandatory for MRD stratification by study design in the AIEOP-BFM ALL 2000 trial, our study included patients with a single informative marker.

Surveillance MRD results were categorized as MRD negative in the absence of specific amplification or amplification within three threshold cycles of the background to exclude false-positive results; as MRD low positive if positivity was less than $5 \times 10^{-4}$ or below the achieved quantitative range (ie, not quantifiable); or as MRD high positive if the positivity was $\geq 5 \times 10^{-4}$, the threshold used in the AIEOP-BFM ALL 2000 study to define HR-MRD levels after induction and consolidation.

\section{RESULTS}

\section{Study Population}

The main features of the 110 study patients are summarized in Table 1. The analysis found a strong association between higher PCR MRD levels at the two AIEOP-BFM ALL 2000 protocol TPs (TP1, day +33; TP2, day +78) and the frequency of MRD positivity at later TPs; patients with favorable genetic features (hyperdiploidy or TEL-AML positivity) and B-cell precursor immunophenotype were less likely (although not significantly) to have MRD positivity at later TPs. Table 2 depicts the outcome of treatment: at a median follow-up time of 9.8 


\begin{tabular}{|c|c|c|c|c|c|c|c|c|}
\hline & & \multicolumn{6}{|c|}{ PCR-MRD } & \multirow[b]{3}{*}{$P$} \\
\hline \multicolumn{2}{|c|}{ Total } & \multicolumn{2}{|c|}{ Negative } & \multicolumn{2}{|c|}{$\begin{array}{l}\text { Low Positive } \\
\left(<5 \times 10^{-4}\right)\end{array}$} & \multicolumn{2}{|c|}{$\begin{array}{l}\text { High Positive } \\
\left(\geq 5 \times 10^{-4}\right)\end{array}$} & \\
\hline No. & $\%$ & No. & $\%$ & No. & $\%$ & No. & $\%$ & \\
\hline 110 & & 81 & 73.6 & 23 & 20.9 & 6 & 5.5 & \\
\hline & & & & & & & & .74 \\
\hline & & & & & & & & 1.0 \\
\hline 93 & 84.6 & 68 & 84.0 & 20 & 87.0 & 5 & 83.3 & \\
\hline 17 & 15.4 & 13 & 16.0 & 3 & 13.0 & 1 & 16.7 & \\
\hline & & & & & & & & .38 \\
\hline 103 & 93.6 & 77 & 95.1 & 21 & 91.3 & 5 & 83.3 & \\
\hline 7 & 6.4 & 4 & 4.9 & 2 & 8.7 & 1 & 16.7 & \\
\hline 50 & 48.5 & 41 & 53.2 & 7 & 35.0 & 2 & 33.3 & \\
\hline 53 & 51.5 & 36 & 46.8 & 13 & 65.0 & 4 & 66.7 & \\
\hline 7 & & 4 & & 3 & & 0 & & \\
\hline & & & & & & & & 1.0 \\
\hline 106 & 96.4 & 78 & 93.8 & 23 & 100.0 & 5 & 83.3 & \\
\hline 4 & 3.6 & 3 & 3.7 & 0 & & 1 & 16.7 & \\
\hline & & & & & & & & .009 \\
\hline 48 & 44.0 & 40 & 49.4 & 8 & 34.8 & 0 & & \\
\hline 42 & 38.5 & 32 & 39.5 & 9 & 39.1 & 1 & 20.0 & \\
\hline 19 & 17.5 & 9 & 11.1 & 6 & 26.1 & 4 & 80.0 & \\
\hline 1 & & 0 & & 0 & & 1 & & \\
\hline & & & & & & & & $<.001$ \\
\hline
\end{tabular}

Table 2. Treatment Outcome of 110 Patients With Childhood ALL, According to the Results of PCR-MRD Monitoring Performed After Induction-Consolidation Treatment

\begin{tabular}{|c|c|c|c|c|c|c|c|c|}
\hline \multirow[b]{3}{*}{ Outcome } & \multirow{2}{*}{\multicolumn{2}{|c|}{ Total }} & \multicolumn{6}{|c|}{ PCR-MRD } \\
\hline & & & \multicolumn{2}{|c|}{ Negative } & \multicolumn{2}{|c|}{$\begin{array}{l}\text { Low Positive } \\
\left(<5 \times 10^{-4}\right)\end{array}$} & \multicolumn{2}{|c|}{$\begin{array}{l}\text { High Positive } \\
\left(\geq 5 \times 10^{-4}\right)\end{array}$} \\
\hline & No. & $\%$ & No. & $\%$ & No. & $\%$ & No. & $\%$ \\
\hline Total No. of patients & 110 & & 81 & & 23 & & 6 & \\
\hline First remission & 87 & 79.1 & 72 & 88.9 & 14 & 60.9 & 1 & 16.7 \\
\hline Relapsed & 23 & 20.9 & 9 & 11.1 & 9 & 39.1 & 5 & 83.3 \\
\hline \multicolumn{9}{|l|}{ Site } \\
\hline $\mathrm{BM}$ isolated & 17 & & 6 & & 6 & & 5 & \\
\hline BM combined & 3 & & 1 & & 2 & & 0 & \\
\hline Extramedullary & 3 & & 2 & & 1 & & 0 & \\
\hline
\end{tabular}

Abbreviations: ALL, acute lymphoblastic leukemia; BM, bone marrow; MRD, minimal residual disease; PCR, polymerase chain reaction. 
years, 23 patients $(20.9 \%)$ had developed a relapse isolated in the BM $(n=17)$, in an extramedullary site $(n=3)$, or in a combination of the two $(\mathrm{n}=3)$, either during or after completion of treatment. All relapses showed the same gene rearrangement already identified at diagnosis. The remaining 87 patients $(79.1 \%)$ were in first CR at the time of last follow-up. The probability of 5-year DFS was $81.8 \%$ (SE, $3.7 \%$ ), with a CIR of $18.2 \%$ (SE, 3.7\%). Their probability of 5-year DFS was not different from that of the whole AIEOP-BFM ALL $2000^{6,10}$ cohort of patients in first CR after induction IA (Appendix Fig A1, online only).

\section{Association Between MRD Results and Outcome}

A total of 588 samples were analyzed, with a median of five samples per patient. The molecular markers analyzed are listed in Appendix Table A1 (online only). Overall, of the 588 samples, $539(91.7 \%)$ were classified as negative, 41 (7\%) as MRD low positive, and eight (1.4\%) as MRD high positive based on at least one marker.

Of the 110 patients included in this study, six (5.5\%) had one or more MRD high-positive results during the observation time, and five of them subsequently relapsed at $1,3,3,8$, and 12 months from the first high-positive result (Table 2 and Appendix Fig A2 [online only]). Thus, the positive predictive value (ie, the probability of relapsing given an MRD high-positive finding after day +78 ) was $83 \%$. A total of 23 patients were classified as low positive at one or more TPs; of those patients, nine relapsed at a median of 18 months after the initial positive finding, with a positive predictive value of $39 \%$; the median follow-up after first positivity of the remaining 14 patients in CR was 9.8 years. A total of 81 patients were MRD negative at all tested TPs; of them, nine relapsed, indicating that the probability of remaining in remission, given that MRD never reached positivity during postinduction treatment (ie, the negative predictive value) was $89 \%$.

The rate of relapse was similar for patients with T-lineage (two of nine patients) or B-lineage (21 of 101) ALL. Among patients with B-lineage ALL, all four patients with high MRD positivity relapsed, accounting for $20 \%$ of all relapses. Of interest, of three patients with B-lineage ALL who developed an isolated extramedullary relapse soon after stopping therapy, one had a single low-positive result at an initial TP and tested negative thereafter, and the other two always tested MRD negative (Appendix Fig A2).

The 5-year CIR was $8.6 \%$ (SE, 3.1\%) for the MRD-negative patients, $34.8 \%$ (SE, 9.9\%) for the patients who had at least one low-positive result, and $83.3 \%$ (SE, 15.2\%) for the patients who had at least one high-positive result (Fig 1). Thus, detection of MRD positivity was significantly related to the risk of relapse $(P<.001)$.

We also evaluated, within the larger subgroup of 96 patients with B-lineage ALL without HR features, whether the MRD profile was predictive of the risk of relapse after adjusting for risk group (SR $v$ IR) in a Cox model. Patients with at least one low-positive (hazard ratio, 4.3; 95\% CI, 1.53 to $12.0 ; P=.006$ ) or high-positive (hazard ratio, $21.2 ; 95 \% \mathrm{CI}, 4.9$ to $91.0 ; P<.001)$ value had a significantly higher risk of relapse.

To assess the added value of short-term versus long-term MRD monitoring, we separately analyzed patients who had MRD positivity $(\mathrm{n}=18)$ or negativity $(\mathrm{n}=86)$ shortly after the induction-consolidation phases (ie, within 4 months after day +78; six patients were not evaluated for early MRD). Of the 18

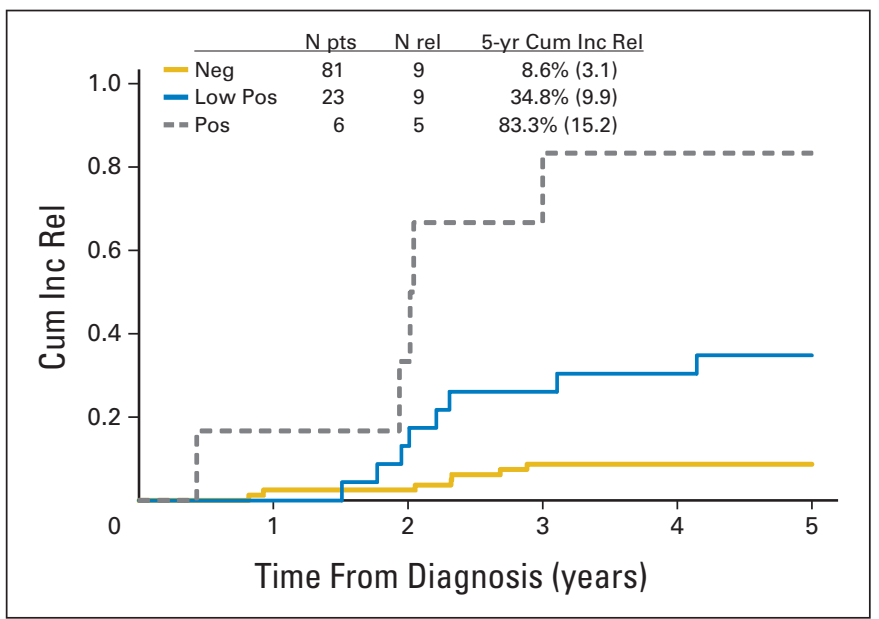

Fig 1. Cumulative incidence of relapse (Cum Inc Rel) in 110 children with acute lymphoblastic leukemia, according to the results of polymerase chain reaction monitoring of minimal residual disease during postinduction treatment. Numbers in parentheses represent SE. Neg, negative; Pos, positive.

patients with early MRD positivity, one relapsed early (HR patient 108); 12 tested repeatedly positive at serial MRD measurements, and five of them relapsed; five subsequently tested MRD negative, but three of them eventually relapsed, too; thus the total number of relapses in this subgroup was nine (50\%). Interestingly, one patient with T-cell ALL (T-ALL) repeatedly tested positive up to the end of treatment; nevertheless, he remained in CR for 12 years after the diagnosis (IR patient 99).

Of the 86 patients who were found to be MRD negative in the 4 months following the induction-consolidation phases, 77 (90\%) remained negative thereafter at all the TPs tested, and nine $(10 \%)$ eventually relapsed; the remaining nine patients instead showed MRD positivity at one (or more) following TPs, and four of them relapsed. Of the six patients who lacked early MRD follow-up, two showed subsequent MRD positivity, and one of them relapsed.

\section{DISCUSSION}

The prognostic value of MRD in childhood ALL has been firmly established by several groups worldwide. In particular, the use of RQ-PCR MRD has been widely documented by the AIEOP-BFM clinical trials as feasible and reproducible. ${ }^{6,10}$ The issue of MRD monitoring at later TPs has been considered since the early 1990s. In the pioneering study by Van Dongen et $\mathrm{al}^{5}$ any MRD positivity at any single TP during first-line treatment was predictive of poor outcome. However, because of the high predictive value of combined MRD results at the first two TPs and because of the rarity of positivity at later TPs, systematic MRD monitoring after induction-consolidation therapy was considered to be not cost-effective. In 2003, the Australian and New Zealand Children's Cancer Study Group reported the results of MRD monitoring at 1 and 2 years from diagnosis, in which patients with positive MRD underwent treatment intensification. ${ }^{25}$ The minority of patients with late MRD positivity had a poor outcome despite 
MRD-directed treatment intensification. Their data allowed the conclusion that systematic MRD monitoring at late TPs could not be recommended. ${ }^{26}$ In our study, we explored the application of extended, prospective PCR MRD monitoring beyond the two TPs used for patient stratification in the AIEOP-BFM studies. The results are strongly predictive of the individual patient's outcome: indeed, patients with high-positive levels $\left(\geq 5 \times 10^{-4}\right.$ ) had a high risk of developing a BM relapse during treatment (five relapses in six patients). This compares with a 39\% relapse rate in patients with a single or repeated MRD low-positive result $\left(<5 \times 10^{-4}\right)$ and an $11 \%$ relapse rate in patients with a negative $\mathrm{MRD}$ profile.

These findings are in keeping with the results obtained by Raff et $\mathrm{al}^{20}$ in the GMALL (German Multicenter Trial for Treatment of Newly Diagnosed Acute Lymphoblastic Leukemia in Adults) 06/99 trial and the GMALL 07/2003 trial in which conversion to MRD positivity during the early postconsolidation phase in adult patients with SR-ALL was highly predictive of subsequent hematologic relapse. Yet our data are only partially in keeping with the recent experience of the German Multicenter Study Group for Adult ALL, documenting that patients in molecular CR after consolidation had a significantly higher probability of both overall survival and DFS compared with patients with evidence of molecular relapse. However, in the adult setting, patients with molecular relapse without transplantation in first CR had a median time to cytologic relapse of 2.6 months from MRD positivity and a probability of continuous complete remission of only $5 \% .^{27}$ In our experience, the probability of continuous complete remission was $60 \%$ in patients $(n=9)$ who tested positive only at later TPs and $50 \%$ in patients $(\mathrm{n}=18)$ who had a molecular positivity early (within 4 months) after induction-consolidation therapy. Although definitely better than the outcome in adult patients, the outcome of this last group is considered inadequate compared with that of the general population of patients with childhood ALL. They account for 16\% (18 of 104) of the patients and can be identified by a relatively low-cost MRD monitoring at a single additional TP within 4 months after induction and consolidation therapy. Monitoring appears to be indicated, especially in patients who test MRD positive at day +78 via PCR: among 21 such patients, 11 remained MRD positive, and five of them relapsed. Early therapeutic interventions with alternative agents and/or with allogeneic HSCT in first CR appears to be indicated. Otherwise, systematic MRD monitoring in 86 patients who were MRD negative within 4 months after induction-consolidation therapy allowed the identification of only nine patients with MRD positivity later on, and four of them relapsed (three close to stopping therapy, one at 7.5 years after diagnosis). It should also be noted that nine relapses occurred in 77 patients $(12 \%)$ in whom no positive signals were detected.

The finding of persistence of MRD-positive results throughout continuation chemotherapy, despite continuous CR in one patient, is puzzling. This patient had IR T-ALL, with adequate initial response (good response to steroid prephase) and low MRD positivity at TP2. Because (by study design) the attending physicians and the family were blinded to the results of the MRD analysis at later TPs, this patient did not receive any additional treatment intensification or extension. MRD measurements after TP2 had never been done in the cooperative AIEOP-BFM group outside this study. In patients with T-ALL and comparable MRD levels in the AIEOP-BFM ALL 2000 study, the probability of remaining relapse-free was $74 \% .{ }^{10}$ The pattern of MRD in this patient might represent the tip of the iceberg if MRD levels fluctuate around and below the threshold of detection; if so, cure for ALL might not necessarily result from complete eradication of the disease. This hypothesis might also explain why patients may occasionally test low positive throughout maintenance therapy without developing relapse, as observed in our study, and it confirms that the definition of molecular relapse remains quite intriguing. ${ }^{18,19}$ In this context, the risk of false MRD positivity should also be considered. This risk is higher for low levels of MRD, and for this reason we applied the EuroMRD guidelines in a more conservative way, as explained in Patients and Methods. Theoretically, false MRD positivity could also occur in the case of persistence of the preleukemic genetic aberration $^{28}$ or of the chromosomal lesion in a nonappropriated target cell. ${ }^{29}$ Our patient 99 was actually monitored by using the SIL-TAL genomic break point marker, in which a putative preleukemic origin of the translocation cannot be ruled out.

Yet the unsolved dilemma remains on whether available technologies are able to define premorphologic relapse at a time interval which may become suitable for useful clinical intervention. It is interesting to remember that, in the past, an attempt to herald leukemia by the peripheral cell blood count did not result in any therapeutic advantage for the children and was thus abandoned. ${ }^{30}$ In addition, it should be considered that systematic MRD monitoring implies a major investment of resources and an important psychological burden on patients and families. These aspects deserve attention when drawing conclusions about the results of protracted MRD monitoring.

In our opinion, our data suggest that additional MRD evaluation within a few months after induction and consolidation therapy may be clinically justified, particularly in patients who did not reach MRD negativity, because it allows identification of a nonnegligible fraction of patients who have a high risk of relapse. This is important in non-HR patients, in whom it is worth investigating whether molecular slow responders may have an advantage from an early intervention. In HR patients, this strategy may provide useful information to define the timing of HSCT or to assess the benefit of specific therapeutic interventions. Systematic monitoring of MRD in patients who reach MRD negativity by the end of induction-consolidation therapy appears to be not justified in our experience.

\section{AUTHORS' DISCLOSURES OF POTENTIAL CONFLICTS OF INTEREST}

The author(s) indicated no potential conflicts of interest.

\section{AUTHOR CONTRIBUTIONS}

Conception and design: Giuseppe Basso

Provision of study materials or patients: Elena Barisone

Collection and assembly of data: Elena Barisone, Katia Polato, Guiseppe

Basso, Eugenia Giraldi

Data analysis and interpretation: Maddalena Paganin, Giulia Fabbri,

Valentino Conter, Giovanni Cazzaniga, Franca Fagioli, Maurizio Arico,

Maria Grazia Valsecchi, Giuseppe Basso

Manuscript writing: All authors

Final approval of manuscript: All authors 


\section{REFERENCES}

1. Pui $\mathrm{CH}$, Mullighan $\mathrm{CG}$, Evans WE, et al: Pediatric acute lymphoblastic leukemia: Where are we going and how do we get there? Blood 120:11651174,2012

2. Bhojwani D, Pui CH: Relapsed childhood acute lymphoblastic leukaemia. Lancet Oncol 14: e205-e217, 2013

3. Tallen G, Ratei R, Mann G, et al: Long-term outcome in children with relapsed acute lymphoblastic leukemia after time-point and site-of-relapse stratification and intensified short-course multidrug chemotherapy: Results of trial ALL-REZ BFM 90. $\mathrm{J}$ Clin Oncol 28:2339-2347, 2010

4. Cavé $H$, van der Werff ten Bosch J, Suciu S, et al: Clinical significance of minimal residual disease in childhood acute lymphoblastic leukemia: European Organization for Research and Treatment of Cancer-Childhood Leukemia Cooperative Group. N Engl J Med 339:591-598, 1998

5. van Dongen JJ, Seriu T, Panzer-Grümayer ER, et al: Prognostic value of minimal residual disease in acute lymphoblastic leukaemia in childhood. Lancet 352:1731-1738, 1998

6. Conter V, Bartram CR, Valsecchi MG, et al: Molecular response to treatment redefines all prognostic factors in children and adolescents with B-cell precursor acute lymphoblastic leukemia: Results in 3184 patients of the AIEOP-BFM ALL 2000 study. Blood 115:3206-3214, 2010

7. Schrappe M: Minimal residual disease: Optimal methods, timing, and clinical relevance for an individual patient. Hematology Am Soc Hematol Educ Program 2012:137-142, 2012

8. Campana D: Minimal residual disease in acute lymphoblastic leukemia. Hematology Am Soc Hematol Educ Program 2010:7-12, 2010

9. Garand R, Beldjord K, Cavé $H$, et al: Flow cytometry and IG/TCR quantitative PCR for minimal residual disease quantitation in acute lymphoblastic leukemia: A French multicenter prospective study on behalf of the FRALLE, EORTC and GRAALL. Leukemia 27:370-376, 2013

10. Schrappe $M$, Valsecchi $M G$, Bartram $C R$, et al: Late MRD response determines relapse risk overall and in subsets of childhood T-cell ALL: Results of the AIEOP-BFM-ALL 2000 study. Blood 118:20772084, 2011
11. Eckert C, Henze G, Seeger K, et al: Use of allogeneic hematopoietic stem-cell transplantation based on minimal residual disease response improves outcomes for children with relapsed acute lymphoblastic leukemia in the intermediate-risk group. J Clin Oncol 31:2736-2742, 2013

12. Denys $B$, van der Sluijs-Gelling AJ, Homburg $C$, et al: Improved flow cytometric detection of minimal residual disease in childhood acute lymphoblastic leukemia. Leukemia 27:635-641, 2013

13. Eckert C, Biondi A, Seeger K, et al: Prognostic value of minimal residual disease in relapsed childhood acute lymphoblastic leukaemia. Lancet 358: 1239-1241, 2001

14. Gaipa G, Cazzaniga G, Valsecchi MG, et al: Time point-dependent concordance of flow cytometry and real-time quantitative polymerase chain reaction for minimal residual disease detection in childhood acute lymphoblastic leukemia. Haematologica 97:1582-1593, 2012

15. Coustan-Smith E, Behm FG, Sanchez J, et al: Immunological detection of minimal residual disease in children with acute lymphoblastic leukaemia. Lancet 351:550-554, 1998

16. Borowitz MJ, Devidas M, Hunger SP, et al: Clinical significance of minimal residual disease in childhood acute lymphoblastic leukemia and its relationship to other prognostic factors: A Children's Oncology Group study. Blood 111:5477-5485, 2008

17. Flohr T, Schrauder A, Cazzaniga G, et al: Minimal residual disease-directed risk stratification using real-time quantitative PCR analysis of immunoglobulin and T-cell receptor gene rearrangements in the international multicenter trial AIEOP-BFM ALL 2000 for childhood acute lymphoblastic leukemia. Leukemia 22:771-782, 2008

18. Pui $\mathrm{CH}$, Campana D: New definition of remission in childhood acute lymphoblastic leukemia. Leukemia 14:783-785, 2000

19. Aricó $M$, Baruchel $A$, Bertrand $Y$, et al: The Seventh International Childhood Acute Lymphoblastic Leukemia Workshop Report: Palermo, Italy, January 29-30, 2005. Leukemia 19:1145-1152, 2005

20. Raff T, Gökbuget N, Lüschen S, et al: Molecular relapse in adult standard-risk ALL patients detected by prospective MRD monitoring during and after maintenance treatment: Data from the GMALL 06/99 and 07/03 trials. Blood 109:910-915, 2007
21. Lo Coco F, Diverio D, Avvisati G, et al: Therapy of molecular relapse in acute promyelocytic leukemia. Blood 94:2225-2229, 1999

22. Pongers-Willemse MJ, Seriu T, Stolz F, et al: Primers and protocols for standardized detection of minimal residual disease in acute lymphoblastic leukemia using immunoglobulin and $\mathrm{T}$ cell receptor gene rearrangements and TAL1 deletions as PCR targets: Report of the BIOMED-1 CONCERTED ACTION-Investigation of minimal residual disease in acute leukaemia. Leukemia 13:110-118, 1999

23. van Dongen JJ, Langerak AW, Brüggemann $M$, et al: Design and standardization of PCR primers and protocols for detection of clonal immunoglobulin and T-cell receptor gene recombinations in suspect lymphoproliferations: Report of the BIOMED-2 Concerted Action BMH4-CT98-3936. Leukemia 17: 2257-2317, 2003

24. van der Velden VH, Cazzaniga G, Schrauder A, et al: Analysis of minimal residual disease by $\mathrm{lg} / \mathrm{TCR}$ gene rearrangements: Guidelines for interpretation of real-time quantitative PCR data. Leukemia 21: 604-611, 2007

25. Marshall GM, Haber M, Kwan E, et al: Importance of minimal residual disease testing during the second year of therapy for children with acute lymphoblastic leukemia. J Clin Oncol 21:704-709, 2003

26. Aricò M, Conter V, Valsecchi MG, et al: Importance of minimal residual disease testing during the second year of disease: Still no answer? J Clin Oncol 21:4463-4464, 2003

27. Gökbuget $N$, Kneba M, Raff $T$, et al: Adult patients with acute lymphoblastic leukemia and molecular failure display a poor prognosis and are candidates for stem cell transplantation and targeted therapies. Blood 120:1868-1876, 2012

28. Hong D, Gupta R, Ancliff P, et al: Initiating and cancer-propagating cells in TEL-AML1-associated childhood leukemia. Science 319:336-339, 2008

29. Zaliova M, Fronkova E, Krejcikova $K$, et al: Quantification of fusion transcript reveals a subgroup with distinct biological properties and predicts relapse in BCR/ABL-positive ALL: Implications for residual disease monitoring. Leukemia 23:944-951, 2009

30. Rubnitz JE, Hijiya N, Zhou Y, et al: Lack of benefit of early detection of relapse after completion of therapy for acute lymphoblastic leukemia. Pediatr Blood Cancer 44:138-141, 2005 


\section{Appendix}

\section{Treatment Protocol}

All patients were given 7 days of prophase therapy, including steroid therapy (prednisone) and one intrathecal dose of methotrexate (MTX), followed by induction protocol IA and induction consolidation protocol IB; on day 8, patients were randomly assigned to continue steroid treatment with either prednisone $\left(60 \mathrm{mg} / \mathrm{m}^{2}\right.$ per day) or dexamethasone $\left(10 \mathrm{mg} / \mathrm{m}^{2}\right.$ per day) until day 28 (the dose of steroid [prednisone or dexamethasone] was tapered at the end of the time interval for full-dose assumption). Standard-risk patients received four courses of high-dose MTX (2 $\mathrm{g} / \mathrm{m}^{2}$; protocol M) and intermediate-risk patients received $5 \mathrm{~g} / \mathrm{m}^{2}$; protocol M), oral 6-mercaptopurine, and intrathecal therapy. At the beginning of the reinduction phase, a second randomization was planned: for standard-risk patients, protocol II versus reduced-intensity protocol III; for intermediate-risk patients, protocol II versus reducedintensity protocol III given twice; for high-risk patients, three blocks of non-cross-resistant drugs followed by protocol III given three times versus protocol II given twice. Maintenance therapy consisted of 6-mercaptopurine once per day and MTX once per week until 24 months from diagnosis. CNS-directed therapy consisted of intrathecal MTX during each treatment phase including continuation unless cranial radiotherapy (dosage by age) was given to the following patients: high-risk patients age 2 years or older, or those with non-highrisk T-ALL and leukocyte count of more than 100,000/ $\mu \mathrm{L}$ at diagnosis, or those with CNS involvement.

\begin{tabular}{|c|c|c|c|c|}
\hline Patient & Marker & Quantitative Range & Sensitive Range & Present in the Relapse \\
\hline 1 & VH3JH4 & $1 \times 10^{-4}$ & $1 \times 10^{-5}$ & $x$ \\
\hline 2 & VH6JH5 & $5 \times 10^{-4}$ & $1 \times 10^{-5}$ & $x$ \\
\hline 3 & VKIIKDE & $5 \times 10^{-4}$ & $1 \times 10^{-5}$ & $x$ \\
\hline 4 & VH1JH4 & $1 \times 10^{-3}$ & $1 \times 10^{-4}$ & $x$ \\
\hline 5 & DH5JH5 & $1 \times 10^{-3}$ & $1 \times 10^{-5}$ & $x$ \\
\hline 6 & VH3JH6 & $5 \times 10^{-4}$ & $1 \times 10^{-5}$ & $x$ \\
\hline 7 & VH1JH6 & $1 \times 10^{-4}$ & $1 \times 10^{-5}$ & Yes \\
\hline 8 & VH1JH6 & $1 \times 10^{-4}$ & $1 \times 10^{-5}$ & Yes* \\
\hline 9 & VH2JH6 & $1 \times 10^{-3}$ & $1 \times 10^{-5}$ & X \\
\hline 10 & VKIIKDE & $1 \times 10^{-3}$ & $1 \times 10^{-5}$ & Yes \\
\hline 11 & VH1JH4 & $5 \times 10^{-4}$ & $1 \times 10^{-4}$ & $x$ \\
\hline 12 & VH3JH5 & $1 \times 10^{-3}$ & $1 \times 10^{-4}$ & $x$ \\
\hline 13 & DH4JH6 & $5 \times 10^{-4}$ & $1 \times 10^{-5}$ & $x$ \\
\hline 14 & VD2DD3 & $1 \times 10^{-4}$ & $1 \times 10^{-4}$ & $x$ \\
\hline 15 & VH4JH4 & $1 \times 10^{-4}$ & $1 \times 10^{-5}$ & $x$ \\
\hline 16 & VH3JH1 & $5 \times 10^{-4}$ & $1 \times 10^{-4}$ & $x$ \\
\hline 17 & VH6JH4 & $5 \times 10^{-4}$ & $1 \times 10^{-5}$ & $x$ \\
\hline $18^{\circ}$ & VH3JH6 & NDt & $1 \times 10^{-4}$ & Yes \\
\hline 19 & VH4JH2 & $1 \times 10^{-4}$ & $1 \times 10^{-5}$ & $x$ \\
\hline 20 & VKIKDE & $1 \times 10^{-4}$ & $1 \times 10^{-5}$ & $x$ \\
\hline 21 & VD2JD1 & $1 \times 10^{-3}$ & $1 \times 10^{-4}$ & $x$ \\
\hline 22 & VH3JH6 & $5 \times 10^{-4}$ & $1 \times 10^{-4}$ & $x$ \\
\hline 23 & VH3JH6 & $1 \times 10^{-4}$ & $1 \times 10^{-5}$ & $x$ \\
\hline 24 & VH3JH4 & $1 \times 10^{-4}$ & $1 \times 10^{-5}$ & $x$ \\
\hline 25 & VH3JH6 & $5 \times 10^{-4}$ & $1 \times 10^{-4}$ & $x$ \\
\hline 26 & VH3JH5 & $1 \times 10^{-4}$ & $1 \times 10^{-5}$ & $x$ \\
\hline 27 & DH6JH4 & $5 \times 10^{-4}$ & $1 \times 10^{-4}$ & $x$ \\
\hline 28 & VH1JH5 & $1 \times 10^{-4}$ & $1 \times 10^{-5}$ & $x$ \\
\hline 29 & VH3JH4 & $1 \times 10^{-3}$ & $1 \times 10^{-5}$ & $x$ \\
\hline 30 & VD2DD3 & $1 \times 10^{-4}$ & $1 \times 10^{-4}$ & $x$ \\
\hline 31 & VH4JH6 & $1 \times 10^{-5}$ & $1 \times 10^{-5}$ & $x$ \\
\hline 32 & VH4JH5 & $1 \times 10^{-4}$ & $1 \times 10^{-5}$ & $x$ \\
\hline 33 & DD2DD3 & $5 \times 10^{-4}$ & $1 \times 10^{-4}$ & $x$ \\
\hline 34 & VH3JH6 & $5 \times 10^{-4}$ & $1 \times 10^{-5}$ & $x$ \\
\hline 35 & VH3JH4 & $1 \times 10^{-4}$ & $1 \times 10^{-5}$ & $x$ \\
\hline 36 & VH3JH4 & $1 \times 10^{-4}$ & $1 \times 10^{-4}$ & $x$ \\
\hline 37 & VH6JH4 & $1 \times 10^{-4}$ & $1 \times 10^{-5}$ & $x$ \\
\hline 38 & VH7JH6 & $1 \times 10^{-3}$ & $1 \times 10^{-4}$ & $x$ \\
\hline 39 & VH3JH6 & $1 \times 10^{-3}$ & $1 \times 10^{-5}$ & $x$ \\
\hline 40 & VH6JH6 & $\begin{array}{l}5 \times 10^{-4} \\
\text { (continued on } f\end{array}$ & $1 \times 10^{-4}$ & $x$ \\
\hline
\end{tabular}




\begin{tabular}{|c|c|c|c|c|}
\hline Patient & Marker & Quantitative Range & Sensitive Range & Present in the Relapse \\
\hline 41 & VH1JH4 & $1 \times 10^{-4}$ & $1 \times 10^{-5}$ & $\mathrm{x}$ \\
\hline 42 & VH1JH4 & $1 \times 10^{-3}$ & $1 \times 10^{-5}$ & X \\
\hline 43 & VKIIIKDE & $1 \times 10^{-4}$ & $1 \times 10^{-5}$ & Yes* \\
\hline 44 & VH3JH6 & $5 \times 10^{-4}$ & $1 \times 10^{-5}$ & $x$ \\
\hline 45 & VH3JH4 & $1 \times 10^{-4}$ & $1 \times 10^{-5}$ & $x$ \\
\hline 46 & VH2JH4 & $1 \times 10^{-3}$ & $1 \times 10^{-5}$ & $x$ \\
\hline 47 & VH4JH4 & $1 \times 10^{-4}$ & $1 \times 10^{-5}$ & Yes \\
\hline 48 & VH1JH6 & $1 \times 10^{-4}$ & $1 \times 10^{-5}$ & $x$ \\
\hline 49 & VH4JH6 & $1 \times 10^{-4}$ & $1 \times 10^{-4}$ & $x$ \\
\hline 50 & VD1JD1 & $1 \times 10^{-3}$ & $1 \times 10^{-5}$ & $x$ \\
\hline 51 & VH4JH6 & $1 \times 10^{-4}$ & $1 \times 10^{-5}$ & $x$ \\
\hline 52 & VH3JH3 & $5 \times 10^{-4}$ & $1 \times 10^{-5}$ & $x$ \\
\hline 53 & VH3JH3 & $1 \times 10^{-4}$ & $1 \times 10^{-5}$ & $x$ \\
\hline 54 & VD2DD3 & $1 \times 10^{-4}$ & $1 \times 10^{-5}$ & $x$ \\
\hline 55 & VH3JH6 & $1 \times 10^{-4}$ & $1 \times 10^{-5}$ & $x$ \\
\hline 56 & VKIKDE & $5 \times 10^{-4}$ & $1 \times 10^{-5}$ & $x$ \\
\hline 57 & VD2DD3 & $1 \times 10^{-3}$ & $1 \times 10^{-5}$ & $x$ \\
\hline 58 & VD2DD3 & $1 \times 10^{-3}$ & $1 \times 10^{-4}$ & $x$ \\
\hline 59 & VH3JH4 & $1 \times 10^{-4}$ & $1 \times 10^{-4}$ & $x$ \\
\hline 60 & VD2DD3 & $5 \times 10^{-4}$ & $1 \times 10^{-5}$ & $x$ \\
\hline 61 & VB6.4JB1.5 & $5 \times 10^{-4}$ & $1 \times 10^{-4}$ & $x$ \\
\hline 62 & VH3JH5 & $1 \times 10^{-3}$ & $1 \times 10^{-4}$ & $x$ \\
\hline 63 & VH4JH4 & $1 \times 10^{-3}$ & $1 \times 10^{-5}$ & Yes \\
\hline 64 & VKIIKDE & $1 \times 10^{-3}$ & $1 \times 10^{-4}$ & $x$ \\
\hline 65 & VH4JH4 & $1 \times 10^{-4}$ & $1 \times 10^{-5}$ & $x$ \\
\hline 66 & VH3JH4 & $5 \times 10^{-4}$ & $1 \times 10^{-5}$ & $x$ \\
\hline 67 & VH3JH4 & $1 \times 10^{-4}$ & $1 \times 10^{-5}$ & $x$ \\
\hline 68 & VD2DD3 & $1 \times 10^{-3}$ & $1 \times 10^{-4}$ & Yes \\
\hline 69 & VH3JH5 & $5 \times 10^{-4}$ & $1 \times 10^{-4}$ & $x$ \\
\hline 70 & VH3JH5 & $1 \times 10^{-3}$ & $1 \times 10^{-4}$ & $x$ \\
\hline 71 & VH2JH6 & $5 \times 10^{-4}$ & $1 \times 10^{-5}$ & $x$ \\
\hline 72 & VH3JH4 & $1 \times 10^{-4}$ & $1 \times 10^{-5}$ & Yes \\
\hline 73 & VKIKDE & $1 \times 10^{-3}$ & $1 \times 10^{-4}$ & $x$ \\
\hline 74 & VH1JH6 & $1 \times 10^{-4}$ & $1 \times 10^{-5}$ & $x$ \\
\hline 75 & VH3JH4 & $1 \times 10^{-3}$ & $1 \times 10^{-4}$ & $x$ \\
\hline 76 & VH5JH6 & $5 \times 10^{-4}$ & $1 \times 10^{-5}$ & $x$ \\
\hline 77 & VH1JH6 & $5 \times 10^{-4}$ & $1 \times 10^{-4}$ & $x$ \\
\hline 78 & DH6JH6 & $1 \times 10^{-5}$ & $1 \times 10^{-5}$ & $x$ \\
\hline 79 & VD2DD3 & $1 \times 10^{-3}$ & $1 \times 10^{-5}$ & Yes \\
\hline 80 & DH3JH6 & $5 \times 10^{-4}$ & $1 \times 10^{-4}$ & Yes \\
\hline 81 & DH6JH4 & $1 \times 10^{-4}$ & $1 \times 10^{-5}$ & $x$ \\
\hline 82 & VD1JD1 & $5 \times 10^{-4}$ & $1 \times 10^{-5}$ & $x$ \\
\hline 83 & DH4JH6 & $5 \times 10^{-4}$ & $1 \times 10^{-5}$ & $x$ \\
\hline 84 & VH3JH6 & $1 \times 10^{-4}$ & $1 \times 10^{-5}$ & $x$ \\
\hline 85 & VH3JH5 & $1 \times 10^{-4}$ & $1 \times 10^{-4}$ & $x$ \\
\hline 86 & VH1JH6 & $1 \times 10^{-4}$ & $1 \times 10^{-5}$ & $x$ \\
\hline 87 & DD2DD3 & $1 \times 10^{-3}$ & $1 \times 10^{-4}$ & $x$ \\
\hline 88 & VH2JH6 & $1 \times 10^{-3}$ & $1 \times 10^{-4}$ & Yes* \\
\hline 89 & VH1JH4 & $5 \times 10^{-4}$ & $1 \times 10^{-4}$ & Yes \\
\hline 90 & VKIIKDE & $1 \times 10^{-3}$ & $1 \times 10^{-4}$ & $x$ \\
\hline 91 & VH6JH5 & $5 \times 10^{-4}$ & $1 \times 10^{-4}$ & Yes \\
\hline 92 & VH3JH6 & $5 \times 10^{-4}$ & $1 \times 10^{-4}$ & Yes \\
\hline 93 & VD1JD1 & $1 \times 10^{-3}$ & $1 \times 10^{-5}$ & $x$ \\
\hline 94 & VD2DD3 & $5 \times 10^{-4}$ & $5 \times 10^{-4}$ & $x$ \\
\hline 95 & DH6JH4 & $1 \times 10^{-3}$ & $1 \times 10^{-4}$ & $x$ \\
\hline 96 & VH1JH2 & $5 \times 10^{-4}$ & $1 \times 10^{-5}$ & $x$ \\
\hline 97 & VD2DD3 & $5 \times 10^{-4}$ & $1 \times 10^{-5}$ & $x$ \\
\hline 98 & VD2DD3 & $5 \times 10^{-4}$ & $1 \times 10^{-4}$ & $x$ \\
\hline \multirow[t]{2}{*}{99} & SILTAL & $5 \times 10^{-4}$ & $1 \times 10^{-5}$ & $x$ \\
\hline & & \multicolumn{2}{|c|}{ (continued on following page) } & \\
\hline
\end{tabular}




\begin{tabular}{|c|c|c|c|c|}
\hline Patient & Marker & Quantitative Range & Sensitive Range & Present in the Relapse \\
\hline 100 & VH3JH6 & $1 \times 10^{-4}$ & $1 \times 10^{-5}$ & Yes \\
\hline 101 & VH1JH4 & $1 \times 10^{-3}$ & $1 \times 10^{-4}$ & Yes \\
\hline 102 & VD2DD3 & $1 \times 10^{-4}$ & $1 \times 10^{-5}$ & $x$ \\
\hline 103 & VH3JH4 & $1 \times 10^{-3}$ & $1 \times 10^{-4}$ & Yes \\
\hline 104 & VH2JH4 & $5 \times 10^{-4}$ & $5 \times 10^{-4}$ & Yesł \\
\hline 105 & VH3JH3 & $5 \times 10^{-4}$ & $1 \times 10^{-5}$ & X \\
\hline 106 & VD2DD3 & $1 \times 10^{-4}$ & $1 \times 10^{-5}$ & $x$ \\
\hline 107 & VG8JG1.3 & $1 \times 10^{-4}$ & $1 \times 10^{-5}$ & Yes \\
\hline 108 & VH6JH4 & $1 \times 10^{-4}$ & $1 \times 10^{-4}$ & Yes \\
\hline 109 & VD1JD1 & $1 \times 10^{-4}$ & $1 \times 10^{-4}$ & Yes \\
\hline 110 & $\mathrm{DH} 7$ & $1 \times 10^{-3}$ & $1 \times 10^{-5}$ & Yes \\
\hline
\end{tabular}

Abbreviations: ND, not determined; $X$, not tested, the patient did not relapse.

* Low positivity in the bone marrow.

†It was not possible to determine the QR for the patient on 7700. The marker was acquired on 7700 Sequence Detection System (Applied Biosystems); we could

not re-analyze the data according to the guidelines ${ }^{24}$ to give the quantitative range so we define only the sensitive range.

¥Patient 104 partially modified the rearrangement; the MRD primer was designed in the conserved N-region between the DH and $\mathrm{JH}$.

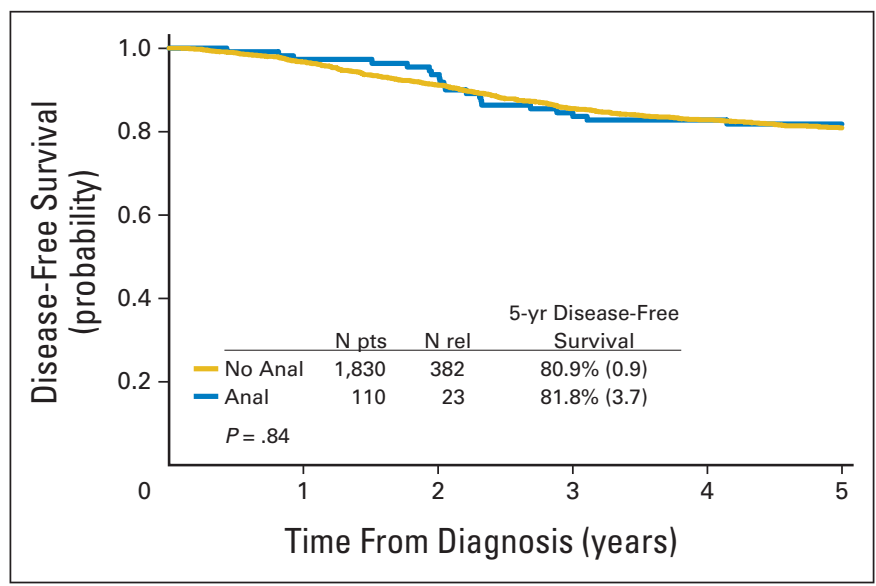

Fig A1. Disease-free survival of the 110 patients selected for this study compared with that of the total study population of the International Collaborative Treatment Protocol for Children and Adolescents With Acute Lymphoblastic Leukemia (AIEOP-BFM ALL 2000) who reached complete remission by the end of phase IA. Our total study population had an outcome that was comparable to that of the overall AIEOP-BFM ALL 2000 study population. Numbers in parentheses represent SE. No Anal, no analysis; pts, patients; rel, relapse. 


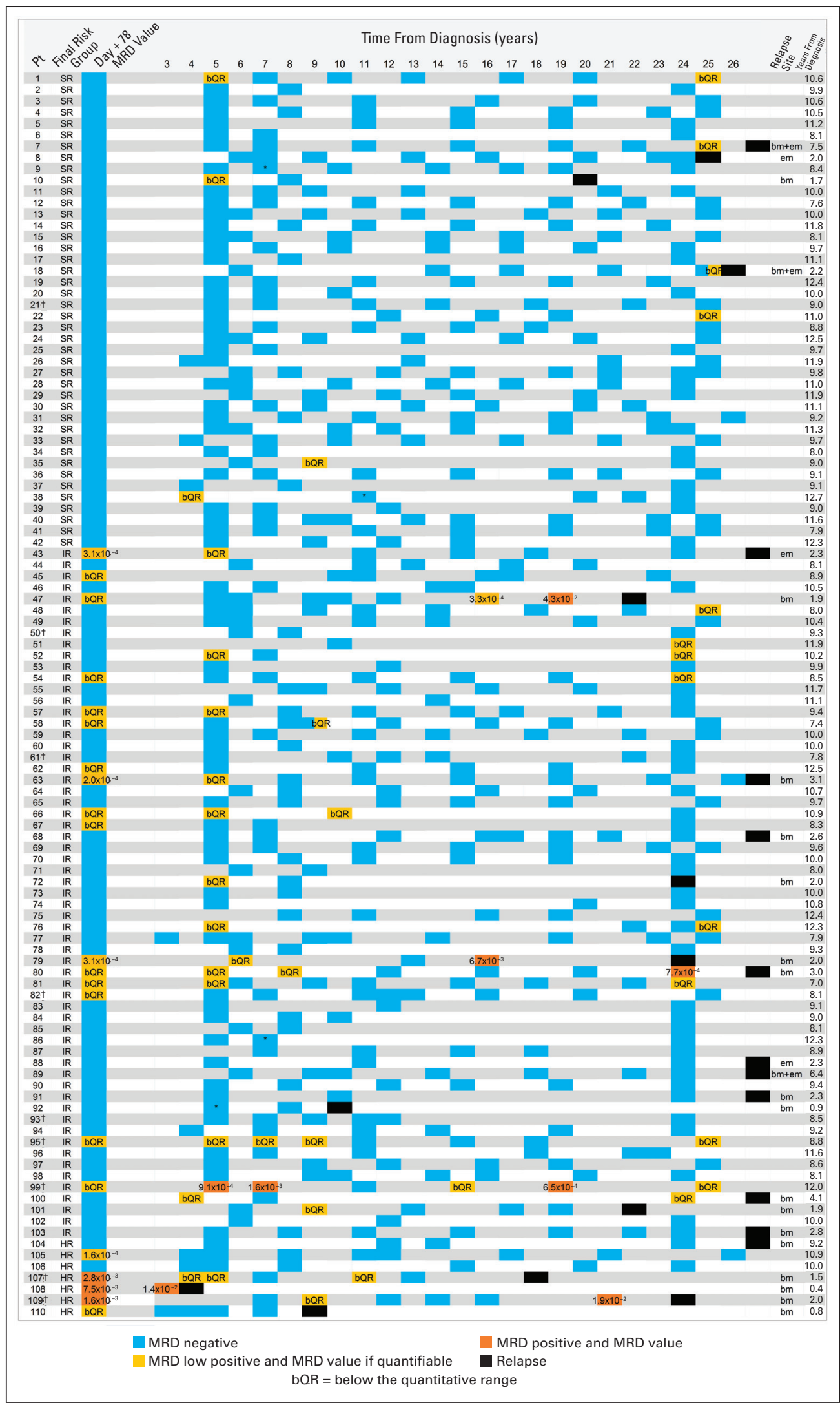

Fig A2. Results of the postinduction monitoring of bone marrow minimal residual disease (MRD) in individual study patients. bm, bone marrow; em, extramedullary; $H R$, high risk; IR, intermediate risk; SR, standard risk. $\left(^{*}\right) 2$ bm samples. ( $\left.\dagger\right) T$ lineage; all other cases were of B lineage. 